\title{
Drosophila Condensin II subunit Chromosome-associated protein D3 regulates cell fate determination through non-cell-autonomous signaling
}

\author{
Lindsey R. Klebanow ${ }^{1}$, Emanuela C. Peshel ${ }^{1}$, Andrew T. Schuster ${ }^{1}$, Kuntal De ${ }^{1}$, Kavitha Sarvepalli ${ }^{1}$, \\ Madeleine E. Lemieux ${ }^{2}$, Jessica J. Lenoir ${ }^{1,3}$, Adrian W. Moore ${ }^{4}$, Jocelyn A. McDonald ${ }^{5}$ and \\ Michelle S. Longworth ${ }^{1, *}$
}

\begin{abstract}
The pattern of the Drosophila melanogaster adult wing is heavily influenced by the expression of proteins that dictate cell fate decisions between intervein and vein during development. dSRF (Blistered) expression in specific regions of the larval wing disc promotes intervein cell fate, whereas EGFR activity promotes vein cell fate. Here, we report that the chromatin-organizing protein CAP-D3 acts to dampen dSRF levels at the anterior/posterior boundary in the larval wing disc, promoting differentiation of cells into the anterior crossvein. CAP-D3 represses KNOT expression in cells immediately adjacent to the anterior/posterior boundary, thus blocking KNOT-mediated repression of EGFR activity and preventing cell death. Maintenance of EGFR activity in these cells depresses dSRF levels in the neighboring anterior crossvein progenitor cells, allowing them to differentiate into vein cells. These findings uncover a novel transcriptional regulatory network influencing Drosophila wing vein development, and are the first to identify a Condensin II subunit as an important regulator of EGFR activity and cell fate determination in vivo.
\end{abstract}

KEY WORDS: dCAP-D3, KNOT, EGFR, Serum response factor, dSRF, Vein development

\section{INTRODUCTION}

Cell fate decisions are often regulated by a combination of factors, including transcriptional regulation imparted though various tissuespecific and developmental stage-specific signaling pathways, as well as positional cues influenced by morphogen gradients. The developing wing discs of the fruit fly Drosophila melanogaster provide an excellent model with which to study mechanisms that control cell fate determination. The adult wing blade pattern includes veins and interveins. There are two types of veins in the wing: longitudinal veins termed L2-L5 and crossveins termed the anterior crossvein (ACV) and the posterior crossvein (PCV).

${ }^{1}$ Department of Cellular and Molecular Medicine, Lerner Research Institute, Cleveland Clinic Foundation, Cleveland, $\mathrm{OH} 44195$, USA. ${ }^{2}$ Bioinfo, Plantagenet, ON K0B 1L0, Canada. 'Department of Molecular Biosciences, Northwestern University, Evanston, IL 60201, USA. ${ }^{4}$ Disease Mechanism Research Core, RIKEN Brain Science Institute, Wako, Saitama 351-0198, Japan. ${ }^{5}$ Division of Biology, Kansas State University, Manhattan, KS 66506, USA.

*Author for correspondence (longwom@ccf.org)

(D) M.S.L., 0000-0002-1313-1777

This is an Open Access article distributed under the terms of the Creative Commons Attribution License (http://creativecommons.org/licenses/by/3.0), which permits unrestricted use, distribution and reproduction in any medium provided that the original work is properly attributed.

Received 1 December 2015; Accepted 3 June 2016
Longitudinal vein primordia appear in the third instar larval stage, and ACV primordia have been reported to appear, although transiently, at this stage as well (Waddington, 1940; Conley et al., 2000). Several signaling pathways regulate wing vein cell fate determination, including EGFR, Hedgehog $(\mathrm{HH})$, DPP and Notch (Sturtevant and Bier, 1995; Sturtevant et al., 1993; Biehs et al., 1998; De Celis, 1997; De Celis et al., 1997; Posakony et al., 1990). EGFR activity drives initial vein-specific gene expression in the larval wing disc, and later maintains vein cell fate specification (through DPP expression) in cells that will become longitudinal veins (Sturtevant et al., 1993; Diaz-Benjumea and Garcia-Bellido, 1990; Guichard et al., 1999; Martin-Blanco et al., 1999; Schnepp et al., 1996; De Celis, 1997). It is known that EGFR signaling is not necessary for the early development of the PCV, but the specific effects of EGFR expression on early development of the ACV and the mechanisms involved are not as well studied.

One way in which EGFR activity controls vein differentiation is through downregulation of the transcription factor Serum response factor (dSRF; also known as Blistered - FlyBase) in longitudinal vein primordia (Roch et al., 1998). dSRF is expressed in third instar larval wing disc cells that are destined to become intervein (Nussbaumer et al., 2000). This expression is then maintained throughout development to eclosure (Montagne et al., 1996). dSRF mutations cause ectopic vein formation, while overexpression of dSRF results in loss of veins, including the ACV (Sturtevant and Bier, 1995; Fristrom et al., 1994; Montagne et al., 1996; Valentine et al., 2014).

Here, we identify a novel role for the Condensin II complex in cell fate determination of third instar larval wing disc cells that will become the ACV. Drosophila Condensin II is composed of four subunits, namely SMC2, SMC4 (Gluon - FlyBase), CAP-H2 and CAP-D3, and functions to organize chromatin throughout the cell cycle. Condensin II is essential for the efficient condensation of chromosomes in mitotic prophase. Condensin II also plays important roles in organizing chromosome territories, in preventing homologous chromosome pairing and in organizing topologically associated domains to regulate transcription (Bauer et al., 2012; Hartl et al., 2008; Li et al., 2015; Joyce et al., 2012). The CAP-D3 subunit of Condensin II regulates the transcription of many genes during the larval and adult stages in the fly, including genes involved in cell fate determination (Longworth et al., 2012). Although Condensin II components have been shown to be necessary for the differentiation of mouse ESCs (Dowen et al., 2013), development of $T$ cells (Rawlings et al., 2011) and differentiation of erythroid progenitors (Xu et al., 2006), a role for these subunits in promoting a choice between two cell fates in vivo has not been reported. 
Here, we show that the cell fate choice to become ACV in the developing wing disc is regulated by CAP-D3 through its ability to maintain EGFR activity in cells immediately anterior to the anterior/ posterior $(\mathrm{A} / \mathrm{P})$ boundary. This prevents cell death, allowing an EGFR-dependent signal to be transmitted to the neighboring cells in the L3-L4 intervein region, which blocks the upregulation of dSRF and prevents those cells from becoming intervein cells. We show that CAP-D3 represses expression of the KNOT transcription factor in the cells anterior to the $\mathrm{A} / \mathrm{P}$ boundary, thus alleviating KNOTmediated repression of EGFR activity. CAP-D3 binds to regions surrounding a knot enhancer and helps to maintain repressive histone marks within the region in S2 cells. These data suggest that CAP-D3/Condensin II may regulate enhancer activity to repress knot transcription and ultimately influence EGFR-mediated signaling to neighboring cells.

\section{RESULTS}

Decreased CAP-D3 expression in cells of the developing wing disc results in loss of the ACV and upregulation of dSRF

Gene ontology analysis of published microarray data comparing gene expression levels in whole, wild-type and Cap-D3 mutant larvae and adults indicated that a significant number of the differentially expressed genes in Cap-D3 mutants are involved in cell fate determination (Longworth and Dyson, 2010). Currently, null alleles of Cap-D3 do not exist. Therefore, in an effort to uncover new roles for CAP-D3 during development, double-stranded RNA (dsRNA) against $C a p-D 3$ was expressed in various tissues and at various stages using different GAL4 drivers (Table 1). The majority of GAL4 drivers used to express Cap-D3 dsRNA resulted in lethality; drivers that induce expression ubiquitously in all tissues caused early lethality prior to the first instar larval stage, whereas tissue-specific drivers more often caused lethality during the pupal stage.

Interestingly, scabrous (sca)-GAL4-driven expression of Cap-D3 dsRNA in specific regions of the developing wing disc, including sensory organ precursor cells (Singson et al., 1994; Mlodzik et al., 1990), resulted in complete or partial loss of the ACV (Fig. 1A). This phenotype was not completely penetrant and was variable in terms of the number of wings per fly that exhibited loss of the ACV. Approximately $50 \%$ of flies expressing Cap-D3 dsRNA driven by sca-GAL4 exhibited loss of the ACV on one or two wings, as compared with control flies expressing GFP dsRNA driven by $s c a$-GAL4 (Fig. 1B).

Flies homozygous for a hypomorphic mutant allele of Cap-D3 (Cap-D3 $\left.3^{c 07081}\right)$ or transheterozygous for this allele in combination with deletions that encompass the Cap-D3 locus $\left(\right.$ Cap-D3 $3^{\text {c07081/Df(2L)Exel6012; }}$ Cap-D3 $\left.3^{\text {07081/Df(2L)BSC169 }}\right)$ do not exhibit loss of the ACV, whereas single copies of these alleles do increase the frequency of ACV loss when expressed together with Cap-D3 dsRNA (Fig. 1B). These results show that further decreasing the levels of CAP-D3 expression in the cells of the developing wing disc significantly impacts the development of the $\mathrm{ACV}$, and also impacts the overall fitness of the organism, since crosses including deficiency alleles exhibited more lethality, with escapers developing wings that lacked ACVs (Fig. 1B). Importantly, overexpression of CAP-D3 protein fused to eGFP at its N-terminus [eGFP-CAP-D3 (Longworth et al., 2012)] together with Cap-D3 dsRNA fully rescued the phenotype in all wings (Fig. 1B). CAP-D3 is a member of the Condensin II complex, which also contains SMC4, SMC2 and CAP-H2. sca-GAL4-driven expression of dsRNA targeting the other Condensin II subunits did not cause loss of the ACV (Fig. S1). However, expression of Cap-D3 dsRNA in flies haploinsufficient for the Condensin II
Table 1. Phenotypic consequences of CAP-D3 deficiency during Drosophila development

\begin{tabular}{|c|c|c|}
\hline GAL4 driver & Area of expression & $\begin{array}{l}\text { Developmental } \\
\text { phenotype at } 25^{\circ} \mathrm{C}\end{array}$ \\
\hline Actin 5C & Ubiquitous & $\begin{array}{l}\text { Early lethal (no first } \\
\text { instar) }\end{array}$ \\
\hline$\alpha T u b 84 B$ & Ubiquitous & $\begin{array}{l}\text { Early lethal (no first } \\
\text { instar) }\end{array}$ \\
\hline $32 B$ & Imaginal discs & $\begin{array}{l}\text { Early lethal (no first } \\
\text { instar) }\end{array}$ \\
\hline patched & $\begin{array}{l}\text { Wing disc and salivary } \\
\text { glands }\end{array}$ & Late lethal (pupal) \\
\hline eyeless & $\begin{array}{l}\text { Eye and other imaginal } \\
\text { discs }\end{array}$ & Late lethal (pupal) \\
\hline engrailed & $\begin{array}{l}\text { Discs, fat body, salivary } \\
\text { glands, cuticle bands }\end{array}$ & Late lethal (pupal) \\
\hline$c 81$ & CNS & Late lethal (pupal) \\
\hline 227 & CNS and epidermis & Late lethal (pupal) \\
\hline $\begin{array}{l}\text { ras2 (Ras64B) } \\
\quad(\text { strong) }\end{array}$ & $\begin{array}{l}\text { Neurons, gut and } \\
\text { proventriculus }\end{array}$ & Late lethal (pupal) \\
\hline sevenless & Eye disc, CNS & Late lethal (pupal) \\
\hline atonal & $\begin{array}{l}\text { Sensory organ } \\
\text { precursors, brain }\end{array}$ & Late lethal (pupal) \\
\hline apterous & $\begin{array}{l}\text { CNS, wing discs and } \\
\text { other imaginal discs }\end{array}$ & Late lethal (pupal) \\
\hline nubbin & $\begin{array}{l}\text { Wing disc, digestive } \\
\text { system and other } \\
\text { imaginal discs }\end{array}$ & Late lethal (pupal) \\
\hline scabrous & $\begin{array}{l}\text { Proneural clusters, } \\
\text { sensory organ } \\
\text { precursors, largely } \\
\text { anterior }\end{array}$ & $\begin{array}{l}\text { Viable, missing } \\
\text { ACV }\end{array}$ \\
\hline $17 a$ & $\begin{array}{l}\text { Glia, cardia and salivary } \\
\text { glands }\end{array}$ & Viable, NP \\
\hline Actin $88 F$ & Neuromuscular junctions & Viable, NP \\
\hline c968a & Larval CNS & Viable, NP \\
\hline elav & $\begin{array}{l}\text { Postmitotic neurons, } \\
\text { transient in embryonic } \\
\text { glial cells }\end{array}$ & Viable, NP \\
\hline
\end{tabular}

Various GAL4 drivers were used to express Cap-D3 dsRNA in varying temporal and tissue-specific patterns. Specifically, ten virgin females harboring an allele for the driver were crossed to ten males harboring the Cap-D3 dsRNA gene and the cross was developed at $25^{\circ} \mathrm{C}$. If any flies expressing both genes (as judged by the absence or presence of balancer chromosomes) developed to eclosure, they were labeled as 'viable' and visible phenotypes were recorded or the absence of phenotype was noted (NP). If lethality occurred, the last observed developmental stage through which the progeny progressed was noted. Independent crosses were repeated three times.

subunit CAP-H2 or the Condensin I/II subunit SMC4 enhanced the frequency of ACV loss (Fig. S2). Expression of Cap-D3 dsRNA in flies haploinsufficient for Condensin I subunits resulted in a slight enhancement of the phenotype, although not as severe as that seen for CAP-H2 (Fig. S2). These results suggest that the development of the ACV depends most directly on CAP-D3 expression, but is strongly influenced by the Condensin II complex as a whole.

To achieve a better understanding of how decreased CAP-D3 expression might lead to loss of the ACV, immunostaining for CAP-D3 protein was performed on third instar larval wing discs expressing Cap-D3 dsRNA or control GFP dsRNA under the control of sca-GAL4 (Fig. 2A, Fig. S3A). Myristolated RFP (mRFP) was also expressed in these discs to label the cells in which protein expression was being driven by GAL4. The results showed that CAP-D3 protein expression is effectively knocked down in cells where $s c a-G A L 4$ drives expression. The efficient knockdown of CAP-D3 by the expressed dsRNA was also demonstrated in third instar larval salivary glands (Fig. S3B). 


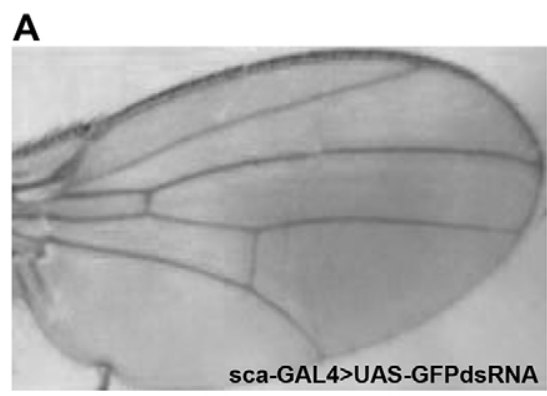

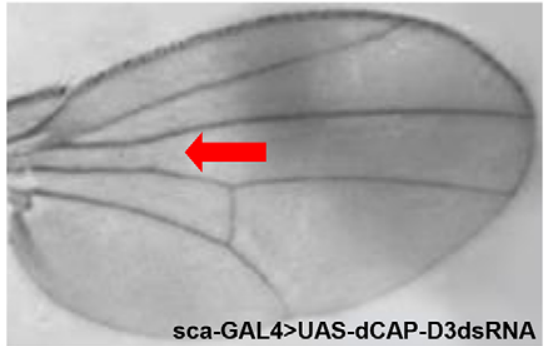

complete loss of ACV

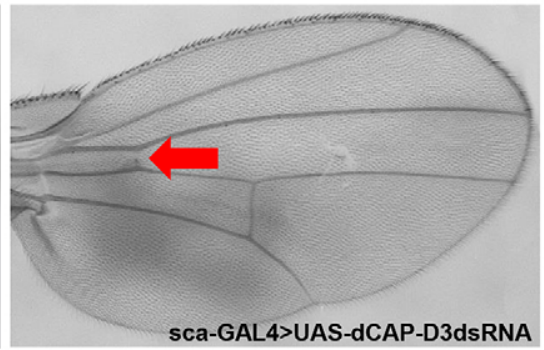

partial loss of ACV
B

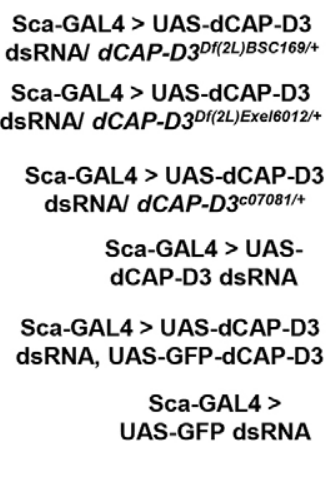

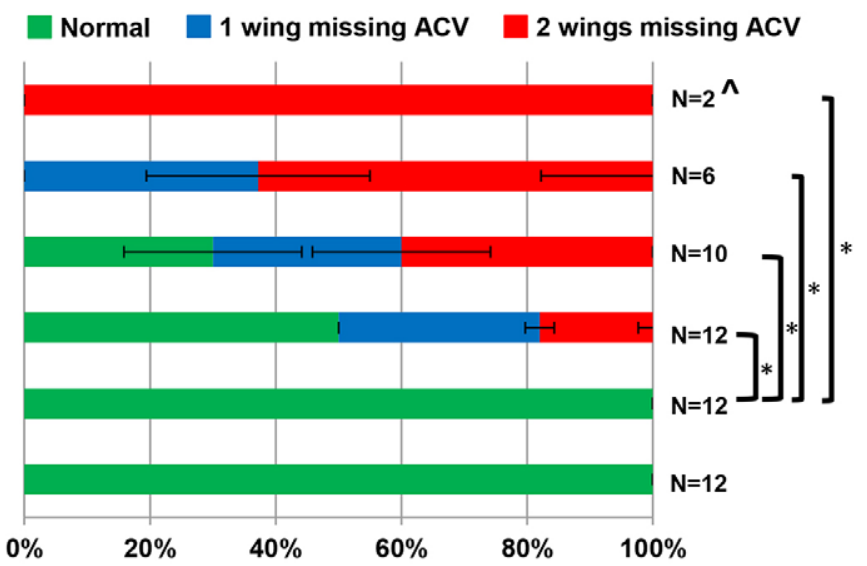

Fig. 1. CAP-D3 deficiency in cells anterior to the anterior/posterior (A/P) border of the developing wing disc results in loss of the anterior crossvein. (A) Adult D. melanogaster wings expressing Cap-D3 dsRNA under the control of sca-GAL4 exhibit complete or partial loss of the anterior crossvein (ACV), as indicated by the red arrow. (B) Frequencies of wings per adult fly that exhibit loss (complete and partial combined) of the ACV. Control flies expressing GFP dsRNA are compared with flies expressing various combinations of alleles that are mutant for Cap-D3, express Cap-D3 dsRNA and/or overexpress CAP-D3. Percentages reflect the average of two crosses with $n$ total flies in comparison to flies expressing Cap-D3 dsRNA alone. ${ }^{\wedge}$ This cross resulted mostly in lethality with analyses performed on $n$ escapers. Error bars indicate s.d. ${ }^{*} P<0.05$ (Fisher's exact test).

Interestingly, in the wing disc, cells deficient for CAP-D3 included cells positioned near the $\mathrm{A} / \mathrm{P}$ boundary, in the region where the ACV is thought to develop (Fig. 2A, Fig. S3A). The $\mathrm{A} / \mathrm{P}$ boundary is maintained largely through $\mathrm{HH}$-mediated regulation of gene expression (Vervoort, 2000). Two HH targets involved in A/P boundary maintenance are Engrailed (EN) and Cubitus interruptus (CI). EN is expressed on the posterior side of the wing disc and $\mathrm{CI}$ is expressed on the anterior side (Vervoort, 2000). Therefore, to determine whether the A/P boundary was disrupted following Cap-D3 dsRNA expression in cells anterior to the A/P boundary, immunostaining for EN and CI was performed separately (Fig. S4). The results demonstrated that normal $\mathrm{EN}$ and $\mathrm{CI}$ protein expression patterns were maintained in Cap-D3 dsRNA-expressing wing discs, as compared with wing discs expressing GFP dsRNA, suggesting that no gross changes to the A/P boundary occur as a result of Cap-D3 dsRNA expression.

Previous work from other labs has shown that dSRF levels in cells at the A/P boundary of the developing wing disc have a significant impact on the development of wing veins, including the ACV (Roch et al., 1998; Montagne et al., 1996; Fristrom et al., 1994). Specifically, overexpression of dSRF leads to loss of the $\mathrm{ACV}$, whereas decreased dSRF expression leads to ectopic wing vein formation between L3 and L4 (Roch et al., 1998). Immunostaining of third instar larval wing discs expressing CapD3 dsRNA revealed a striking upregulation of dSRF in cells at the $\mathrm{A} / \mathrm{P}$ boundary that were positioned immediately posterior to the
mRFP-labeled cells expressing Cap-D3 dsRNA (Fig. 2B,C, compare yellow arrows). This upregulation was abrogated in wing discs that express eGFP-CAP-D3 in addition to Cap-D3 dsRNA, suggesting that the increased dSRF levels were caused by CAP-D3 deficiency and were not due to an off-target effect of RNAi (Fig. 2D).

If the loss of the ACV were due to the increased levels of dSRF in cells posterior to Cap-D3 dsRNA-expressing cells, then knocking down the levels of dSRF in those cells should rescue ACV development. To test this, sca-GAL4 was used to drive Cap-D3 dsRNA in flies heterozygous for a mutant allele of $\operatorname{dSRF}\left(b s^{3}\right)$ previously shown to exhibit loss-of-function phenotypes similar to other $d S R F$ alleles (Donlea et al., 2009) (Fig. S5). The results showed that expression of $b s^{3}$ did, in fact, suppress the loss of the $\mathrm{ACV}$. These flies, however, also expressed a mutant plexus $\left(p x^{l}\right)$ allele, and PX has been shown to regulate the expression of many genes involved in wing vein differentiation; $p x$ mutation increases rhomboid (rho) expression, which then results in decreased dSRF expression in specific regions of the wing disc (Garcia-Bellido and De Celis, 1992; Matakatsu et al., 1999). Therefore, Cap-D3 dsRNA was expressed in the background of the mutant $p x^{1}$ allele by itself, but results demonstrated a slight enhancement of phenotype as compared with Cap-D3 dsRNA expression alone (Fig. S5). Taken together, these data suggest that knockdown of CAP-D3 expression in cells immediately anterior to the $\mathrm{A} / \mathrm{P}$ boundary causes an upregulation of dSRF that leads to loss of the ACV later in development. 
A
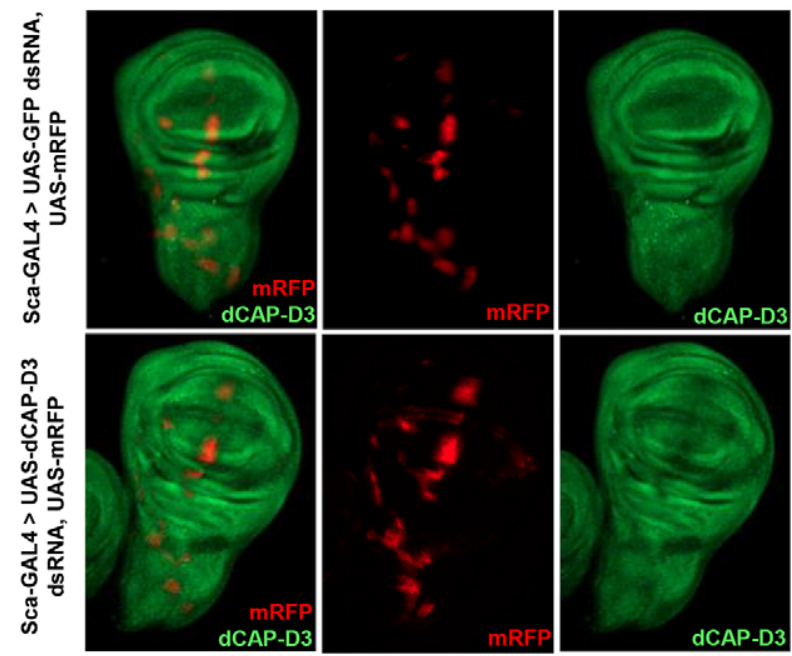

B
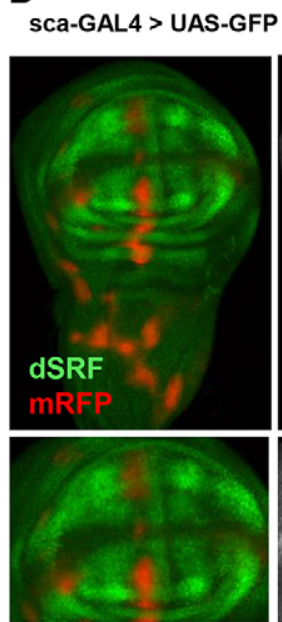

C

sca-GAL4 > UAS-dCAP-D3 dsRNA, UAS-mRFP

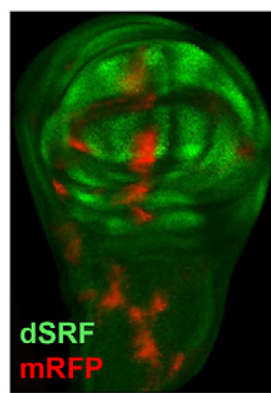

dSRF
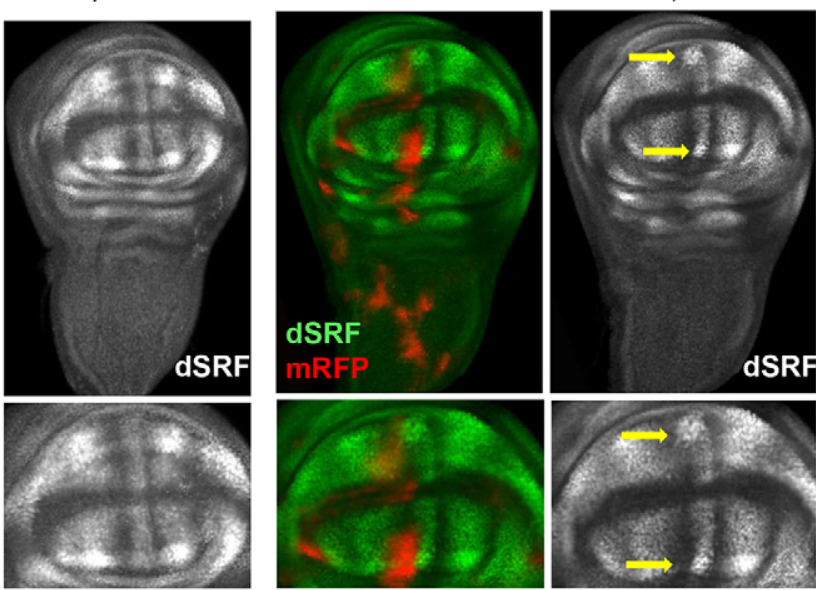

D SCa-GAL4 > UAS-dCAP-D3 dsRNA, UAS-GFP-dCAP-D3

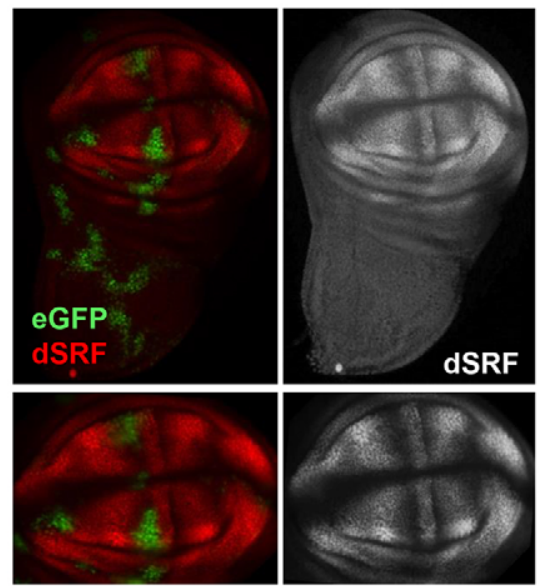

Fig. 2. dSRF expression is increased in cells at the A/P boundary that border CAP-D3-deficient cells in the developing wing disc. (A) Immunostaining for CAP-D3 (green) in third instar larval wing discs expressing mRFP to mark cells in which sca-GAL4 drives expression. Discs expressing Cap-D3 dsRNA show loss of staining in areas expressing mRFP (bottom row) as compared with control wing discs expressing GFP dsRNA (top row). (B) Immunostaining for dSRF (green) in third instar larval wing discs expressing GFP dsRNA reveals a normal staining pattern for dSRF. (C) Immunostaining for dSRF (green) in wing discs expressing Cap-D3 dsRNA shows upregulation of dSRF at the outer edges of both the dorsal and ventral compartments of the pouch, between the L3 and L4 proveins in cells adjacent to those that express mRFP (arrows). (D) Immunostaining for dSRF (red) in wing discs expressing both Cap-D3 dsRNA and eGFPtagged CAP-D3 (green) shows rescue of dSRF levels in A/P boundary cells. Discs shown are representative of three experiments on at least five individual larvae per experiment.

\section{CAP-D3 deficiency results in decreased EGFR activity}

EGF signaling plays a major role in wing vein patterning. EGFR has been shown to repress dSRF levels in the larval wing disc, and loss of EGFR activity results in loss of veins including the ACV (Roch et al., 1998; Price et al., 1989). Since EGFR activation in Drosophila results in phosphorylation of MAPK (ERK) at two specific residues (dpERK), and many of the dpERK staining patterns in Drosophila imaginal discs (including wing discs) correlate with EGFR activation, immunostaining for dpERK is considered to be an effective readout of EGFR activity in the fly (Gabay et al., 1997). EGFR activity was therefore indirectly measured in Cap-D3 dsRNA-expressing cells in the wing disc through immunofluorescence analysis of dpERK. The results demonstrated that control GFP dsRNA-expressing cells (Fig. 3A, top row, marked with mRFP) exhibit high levels of dpERK staining (green), whereas Cap-D3 dsRNA-expressing cells exhibit reduced dpERK staining (Fig. 3A, bottom row). Restoration of EGFR activity through overexpression of EGFR in the Cap-D3 dsRNA-expressing cells in these discs rescued the increased levels of dSRF present in the neighboring $\mathrm{A} / \mathrm{P}$ boundary cells (Fig. 3B, compare middle and left panels). Overexpression of EGFR in cells expressing control GFP dsRNA, however, had no effect on dSRF levels (Fig. 3B, compare right panel with Fig. 2B). In line with these results, overexpression of EGFR in these discs almost completely suppressed the loss-of-ACV phenotype caused by CAP-D3 depletion (Fig. 3C). Additionally, overexpression of secreted forms of various EGFR ligands (Spitz, Gurken, Vein) or expression of dsRNA against an inhibitor of EGFR signaling, Argos, increased lethality in the Cap-D3 dsRNA-expressing progeny, but rescued ACV development in eclosed flies that escaped lethality (Fig. 3C). Overexpression of a dominantnegative form of EGFR in Cap-D3 dsRNA-expressing flies resulted in complete lethality, thus making it impossible to evaluate its effects on ACV development (data not shown). These results indicate that decreased levels of CAP-D3 
A

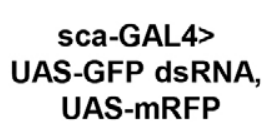
Sca-GAL4>

UAS-GFP dsRNA UAS-mRFP

sca-GAL4>

UAS-dCAP-D3 dsRNA, UAS-mRFP
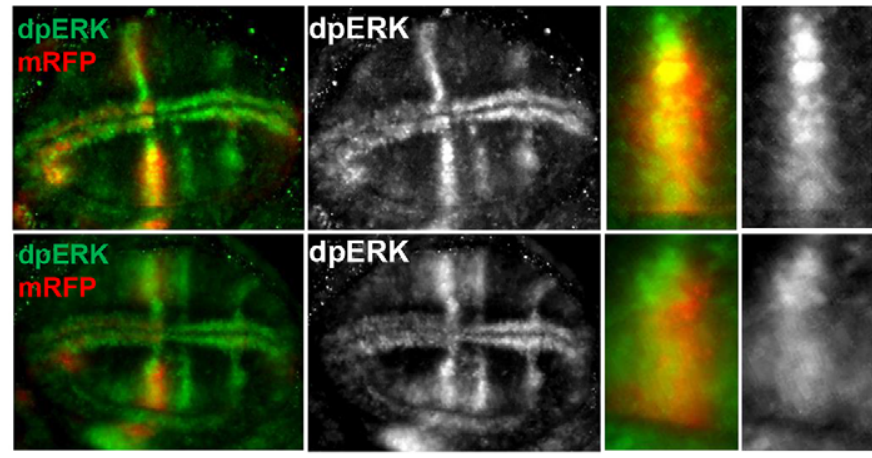

B
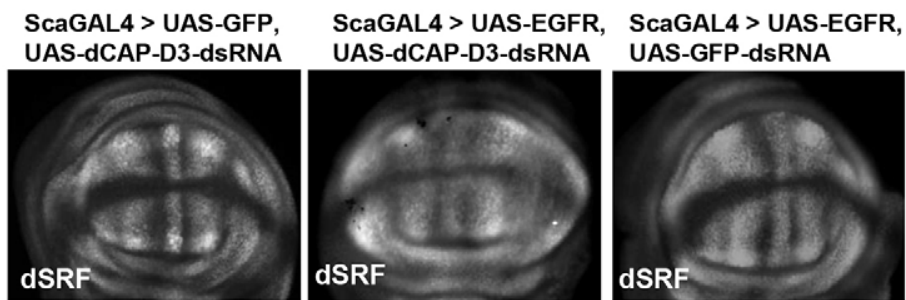

C

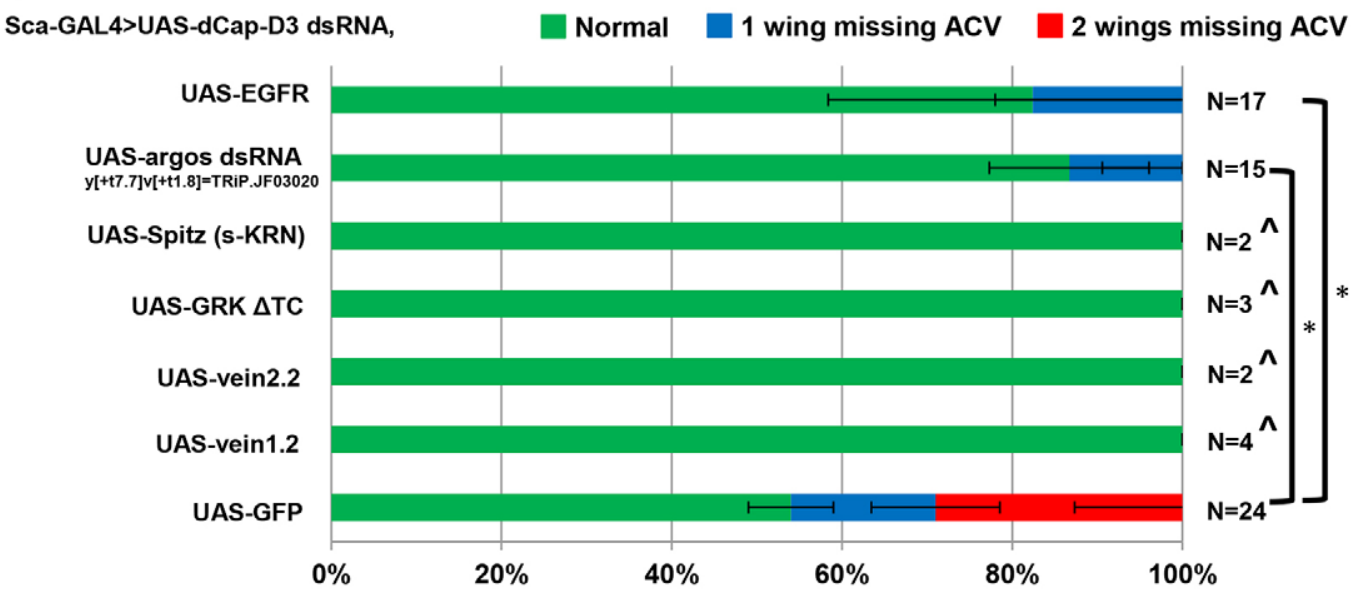

Fig. 3. Increased EGFR activity in CAP-D3-deficient cells decreases dSRF expression at the A/P boundary and rescues ACV development. (A) Immunostaining for dpERK (green) in wing disc cells expressing GFP dsRNA (top row) or Cap-D3 dsRNA (bottom row) driven by sca-GAL4 and marked with mRFP (red) shows lower levels of dpERK in CAP-D3-deficient cells anterior to the A/P boundary. The four panels to the right are magnified images of the staining pattern in the dorsal half of the L3 provein. (B) Immunostaining for dSRF in wing discs expressing Cap-D3 dsRNA compared with those expressing both Cap-D3 dsRNA and UAS-EGFR (left versus middle panels) and compared with discs expressing just UAS-EGFR (right panel) under the control of scaGAL4. Discs shown are representative of three experiments on at least five individual larvae per experiment. (C) Frequencies of wings per adult fly that exhibit loss of the ACV in flies expressing sca-GAL4-driven Cap-D3 dsRNA alone or in combination with UAS-EGFR or regulators of EGFR activity. ${ }^{*} P<0.05$ (Fisher's exact test), in comparison to flies expressing Cap-D3 dsRNA and UAS-GFP. Error bars indicate s.d. ^ This cross resulted mostly in lethality with analyses performed on $n$ escapers.

expression in cells adjacent to the $\mathrm{A} / \mathrm{P}$ boundary result in decreased EGFR activity, which leads to dSRF upregulation and the change to an intervein cell fate decision in the neighboring cells.

\section{KNOT, a repressor of EGFR activity and activator of dSRF expression, is upregulated in CAP-D3-deficient cells}

KNOT is a helix-loop-helix transcription factor of the COE (Collier/ Olf1/EBF) family of transcription factors that has roles in the patterning and development of a number of tissues in the fly. Importantly, KNOT has been shown by several groups to promote intervein cell fate decisions in the wing, largely through its abilities to repress EGFR activity and upregulate dSRF levels (Vervoort et al., 1999; Mohler et al., 2000). Interestingly, in situ hybridization experiments in larval wing discs expressing Cap-D3 dsRNA under the control of $s c a$-GAL4, using an antisense probe to detect knot transcript levels, revealed that knot transcription in third instar larval wing discs is increased in the cells immediately anterior to the $\mathrm{A} / \mathrm{P}$ boundary, as compared with control wing discs expressing GFP dsRNA (Fig. 4A, arrowheads). In situ experiments using a control sense probe did not detect any signal (data not shown). Furthermore, KNOT protein is similarly increased in CAP-D3deficient cells anterior to the $\mathrm{A} / \mathrm{P}$ boundary, as evidenced by immunofluorescence analyses (Fig. 4B, arrowheads). This increase in KNOT protein in Cap-D3 dsRNA-expressing cells was quantified by immunostaining for EN to mark the posterior border, followed by measurement of the spread of KNOT protein staining on the dorsal and ventral edges of the wing pouch, in the 
A

$$
\begin{gathered}
\text { sca-GAL4> } \\
\text { UAS- } \\
\text { GFPdsRNA }
\end{gathered}
$$
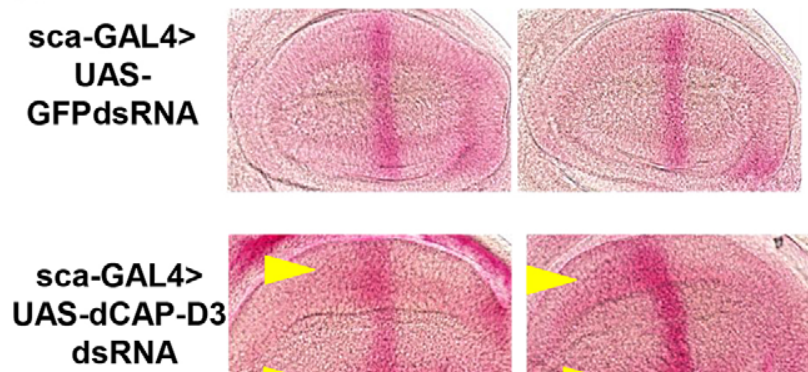

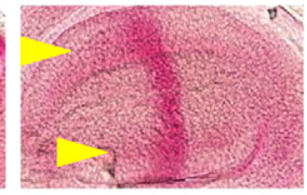

B

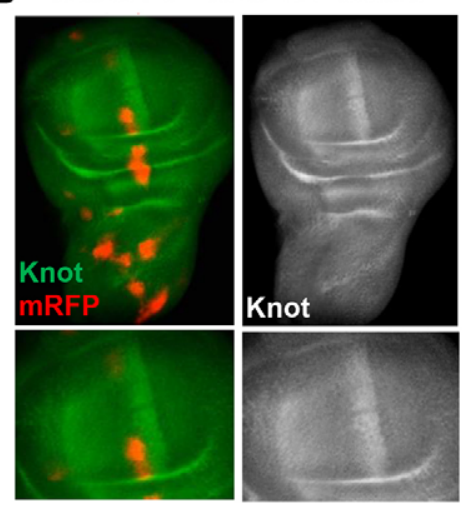

sca-GAL4 > UAS-dCAP-D3 dsRNA
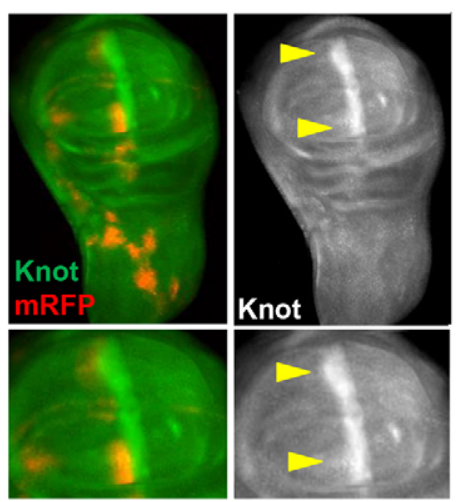

C

Sca-GAL4 > UAS-dCAP-D3dsRNA, kn[col-1]

Sca-GAL4 > UAS-dCAP-D3dsRNA, UAS-knotdsRNAy[+t7.7]v[+t1.8]=TRiP.JF02206

Sca-GAL4 > UAS-dCAP-D3-dsRNA, UAS-knot-dsRNA ${ }^{K K 114322}$

Sca-GAL4 > UAS-dCAP-D3dsRNA, kn[1]

Sca-GAL4 > UASdCAP-D3-dsRNA

Sca-GAL4 > UASGFP-dsRNA

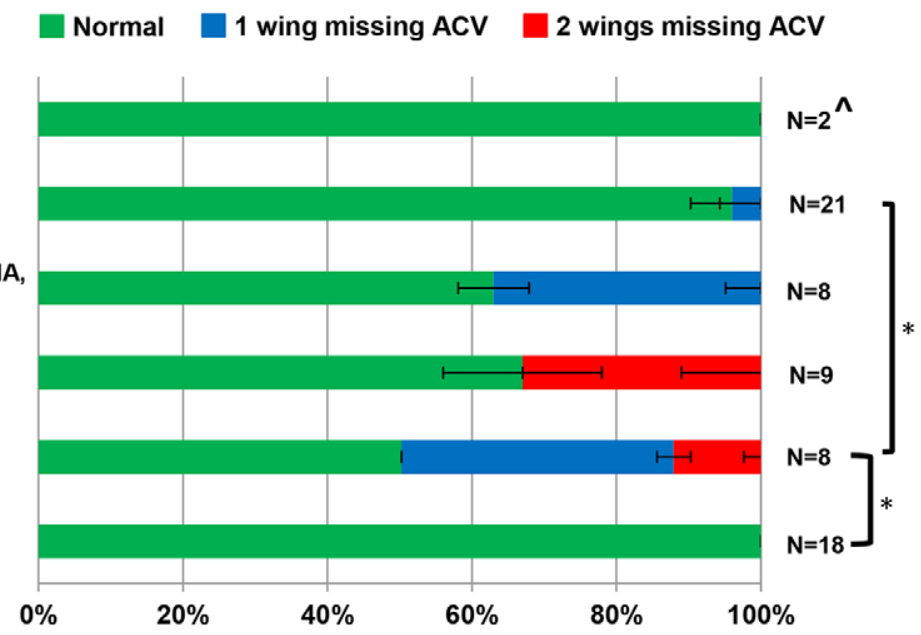

D dSRF

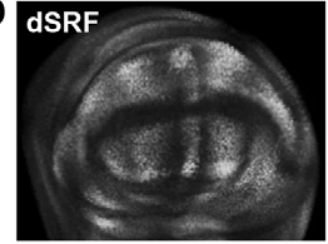

ScaGAL4 > UAS-GFP dsRNA UAS-dCAP-D3-dsRNA

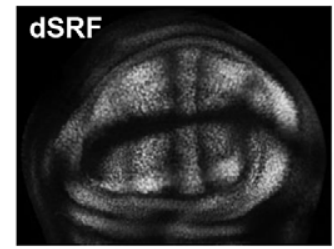

ScaGAL4 > UAS-knot dsRNAJF02206, UAS-dCAP-D3-dsRNA

Fig. 4. CAP-D3-mediated repression of $k$ not transcription is necessary for maintaining low levels of dSRF at the A/P boundary. (A) RNA in situ

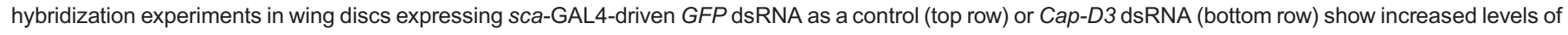
knot transcripts in cells anterior to the A/P boundary (arrowheads). (B) Immunostaining for KNOT in wing disc cells expressing GFP dsRNA (left panels) or Cap-D3 dsRNA (right panels) shows increased KNOT protein levels in cells expressing Cap-D3 dsRNA as marked by mRFP (red) and the arrowheads. (C) Frequencies of wings per adult fly that exhibit loss of the ACV in flies expressing sca-GAL4-driven Cap-D3 dsRNA alone or in combination with knot dsRNAs or mutant knot alleles. Percentages reflect the average of two crosses with $n$ total flies. ${ }^{*} P<0.05$ (Fisher's exact test), in comparison to flies expressing Cap- $D 3$ dsRNA alone. Error bars indicate s.d. ^This cross resulted mostly in lethality with analyses performed on $n$ escapers. (D) Immunostaining for dSRF in wing discs expressing either Cap-D3 dsRNA and GFP dsRNA (top panel) or Cap-D3 dsRNA and knot dsRNA (bottom panel) demonstrates lower levels of dSRF in the Cap-D3/knot dsRNA-expressing discs. Discs shown are representative of three experiments on at least five individual larvae per experiment.

areas where $s c a$-GAL4 drives expression (Fig. S6A, arrows). The distance between the last cell expressing $\mathrm{EN}$ in the posterior compartment and the last cell expressing KNOT in the anterior compartment was measured in the regions at the distalmost portions of the pouch, and then divided by the same measurement taken in the middle of the pouch at the regions on each side of the dorsal/ ventral boundary, to control for changes in disc shape. Results showed a significant increase in the spread of KNOT staining anterior to the $\mathrm{A} / \mathrm{P}$ boundary on the dorsal half of wing discs expressing Cap-D3 dsRNA (Fig. S6B). There was also an average increase in KNOT staining anterior to the A/P boundary on the ventral half of these discs, but it was more variable between discs, and therefore not as statistically significant (Fig. S6C).

To test whether this upregulation of KNOT in CAP-D3-deficient cells anterior to the $\mathrm{A} / \mathrm{P}$ boundary might be responsible for increased dSRF levels in the neighboring cells and the concomitant loss of the ACV, sca-GAL4 was used to drive Cap-D3 dsRNA in combination with knot dsRNA or in flies that were heterozygous for a hypomorphic allele of knot, $k n$ [1], or the amorphic allele $k n$ [col-1] (Fig. 4C). Expression of Cap-D3 dsRNA in cells that also expressed the $k n$ [col-1] allele resulted in complete rescue of ACV development, although the number of progeny that eclosed was drastically reduced. Expression of knot dsRNA or the $k n[1]$ allele also suppressed the loss-of-ACV phenotype, although not to the same extent as the $k n$ [col-1] allele. Co-expression of Cap-D3 dsRNA and knot dsRNA, which suppressed the loss-of-ACV phenotype to the greatest extent (knot dsRNA $A^{y[+t 7.7] v[+t 1.8]=T R i P . J F 02206}$ ), also depressed dSRF in cells in the A/P boundary to levels seen in control wing discs expressing GFP dsRNA (Fig. 4D).

\section{CAP-D3 binds to DNA surrounding the KN01 enhancer in the knot locus}

The expression of knot in the developing wing disc is influenced by many signaling pathways, but transcription in the wing pouch of the third instar larval wing disc is directly controlled by two different enhancers (Hersh and Carroll, 2005; McKay and Lieb, 2013). The more recently identified KN01 enhancer seems to be more active in the pouch, and is located in the fourth intron of the gene (McKay and Lieb, 2013). Since our data suggested that CAP-D3 represses knot transcription, we tested whether it does so directly in S2 cells. 
A

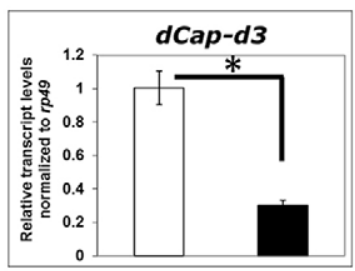

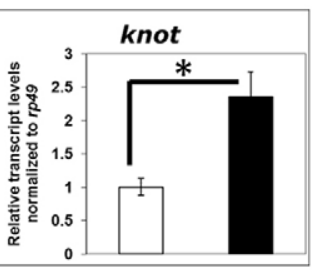

\section{$\square=$ Control dsRNA \\ $=$ dCAP-D3 dsRNA}

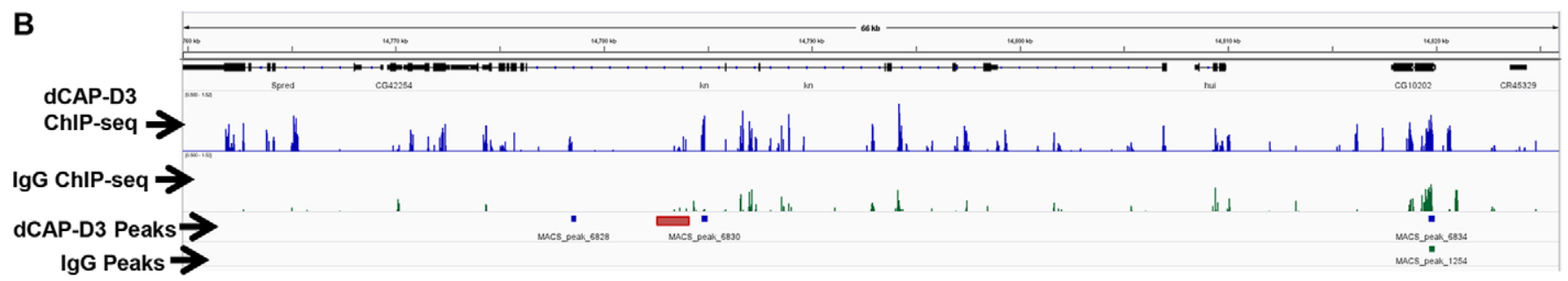

C
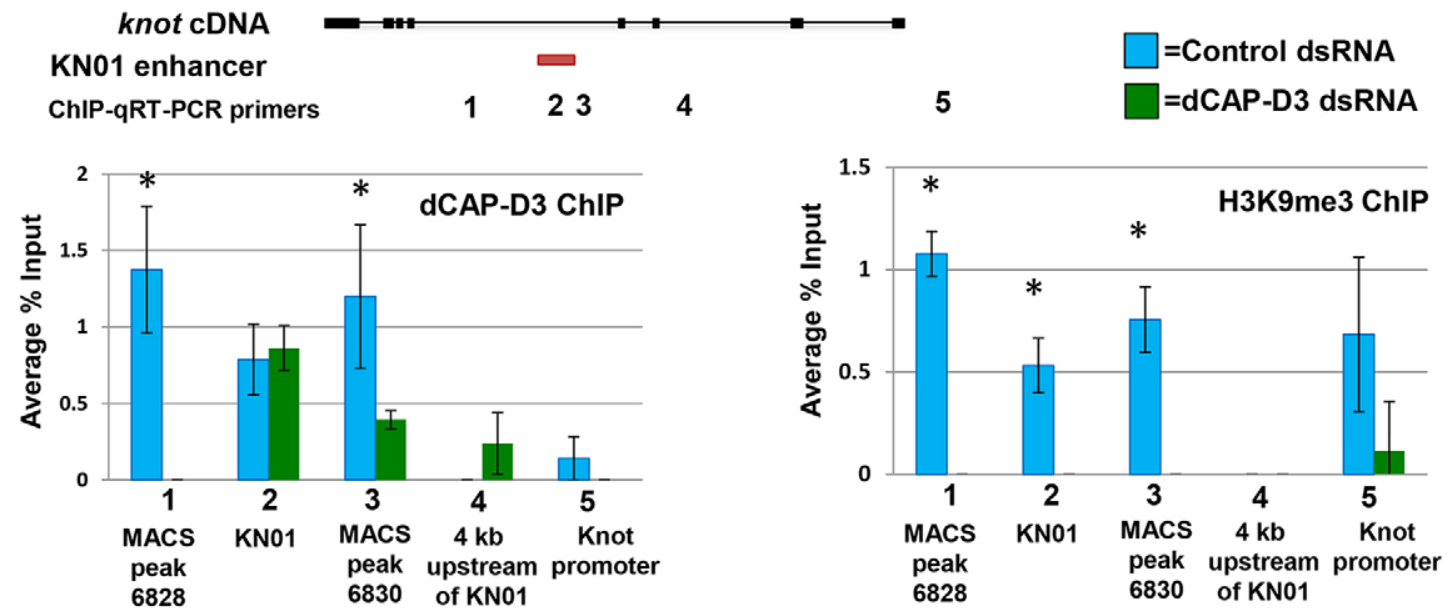

Fig. 5. CAP-D3 binds the region encompassing the knot enhancer KN01 and represses knot transcription in S2 cells. (A) qRT-PCR analysis of Cap-D3 and knot transcript levels in S2 cells shows that Cap-D3 dsRNA treatment decreases Cap-D3 transcripts and increases knot transcripts in comparison to treatment with control (T7) dsRNA. Data are the average of three experiments. (B) Depiction of ChIP-seq results at the knot locus, showing significant CAP-D3 peaks (blue) not found in the set of IgG ChIP-seq peaks (green). All peaks were first normalized to input and significant peaks were identified by MACS peak finder (bottom two rows, where boxes indicate a significant peak). (C) ChIP for CAP-D3 (left) or H3K9me3 (right) was performed in S2 cells treated with control or Cap-D3 dsRNA and analyzed by qRT-PCR. The location of the KN01 enhancer is depicted by a red box. Sequences targeted by primer sets for the ChIP-qRT-PCR experiments are indicated (1-5). ${ }^{*} P<0.05$, Student's unpaired $t$-test; error bars indicate s.d.

qRT-PCR analysis demonstrated that decreased Cap-D3 levels in S2 cells expressing Cap-D3 dsRNA did indeed result in upregulation of knot transcripts, similar to observations in the wing disc (Fig. 5A). These analyses also revealed that $d S R F$ levels remain unchanged in Cap-D3 dsRNA-expressing S2 cells (Fig. S7). This is not surprising, however, given that EGFR protein is not expressed in S2 cells (Schweitzer et al., 1995) and therefore does not act to repress $d S R F$ expression, thus rendering knot upregulation irrelevant.

Chromatin immunoprecipitation for CAP-D3 combined with high-throughput sequencing (ChIP-seq) was next performed in $\mathrm{S} 2$ cells and compared with ChIP-seq for IgG as a control, following normalization of both data sets to the input signal. In total, 9059 binding sites $\left(P=1 \times 10^{-10}\right)$ were identified for CAP-D3 (see GEO accession GSE74413). Previously, it was shown that CAP-D3 orthologs in other organisms bind primarily to gene promoters (Kranz et al., 2013). However, the majority of CAP-D3 binding sites in S2 cells were not within gene promoters, with more than $50 \%$ of the binding sites positioned within exons and introns (Fig. S8).
Upon further inspection of the CAP-D3 ChIP-seq data, it was determined that two significant CAP-D3 binding sites flank the KN01 enhancer sequence (Fig. 5B). To confirm that these sites are bound by CAP-D3 in S2 cells, ChIP with CAP-D3 antibody and qRT-PCR analyses using primers that spanned the knot locus (including the potential CAP-D3 binding sites) were performed in control or Cap-D3 dsRNA-treated cells (Fig. 5C, left panel). Results confirmed that CAP-D3 binding to both of the identified sites (MACS peaks 6828 and 6830) was significantly depleted in Cap-D3 dsRNA-treated cells (Fig. 5C). Interestingly, examination of the top 1000 peaks found outside promoters for common CAP-D3 binding motifs (using the MEME-ChIP web server) revealed that nine motifs were significantly enriched at CAP-D3 binding sites and that one motif, $\mathrm{AA}(\mathrm{G} / \mathrm{A}) \mathrm{TGG}$, is present in the confirmed CAP-D3 binding site corresponding to MACS peak 6830 (sequence not shown).

Given that CAP-D3 has been shown to flank and play a role in the maintenance of repressive chromatin marks of other genes that it transcriptionally represses (Schuster et al., 2013), ChIP analyses for the histone $\mathrm{H} 3$ trimethylated lysine 9 (H3K9me3) repressive mark 
were performed in control and Cap-D3 dsRNA-treated cells. Results showed that $\mathrm{H} 3 \mathrm{~K} 9 \mathrm{me} 3$ is present in the region bound by CAP-D3, which surrounds and encompasses the KN01 enhancer (Fig. 5C, right panel). Furthermore, H3K9me3 marks are significantly depleted at this region in Cap-D3 dsRNA-treated cells, suggesting that CAP-D3 might act to maintain this repressive chromatin mark within the region, potentially promoting a more closed chromatin configuration that might prevent interaction of the KN01 enhancer with the promoter. Finally, ChIP analyses for histone $\mathrm{H} 3$ acetylated at lysine 27 (H3K27ac, a mark found at active enhancers) demonstrated no significant changes in distribution within the region in Cap-D3 dsRNA-treated cells, suggesting that CAP-D3 is not necessary for maintaining H3K27ac at KN01 (Fig. S9). Together, these data suggest that CAP-D3 may directly repress transcription of the knot gene by binding to sites that flank the KN01 enhancer and maintaining repressive chromatin marks within the region.

\section{CAP-D3 represses knot and maintains EGFR activity to prevent cell death and promote non-cell-autonomous repression of dSRF}

EGFR activity is important for preventing cell death in a number of organisms and tissues (Doroquez and Rebay, 2006; Bergmann et al., 1998). Interestingly, sca-GAL4-driven expression of Cap-D3 dsRNA resulted in a significant decrease in the distance between the outer edges of L3 and L4 in the adult wing compared with the GFP dsRNA control [27.41 \pm 3.71 (s.d.) versus $28.82 \pm 2.94 ; n=44$ each; $P<0.05$, Student's unpaired $t$-test]. This indicated that cell death might occur earlier in development in some of the cells in this area. To further examine whether the role of CAP-D3 in promoting EGFR activity is also necessary to prevent cell death, Cap-D3 dsRNA was expressed using the $s c a$-GAL4 driver and wing discs were immunostained for dSRF and DCP-1 (Death caspase-1), a Drosophila member of the caspase family of ICE/CED-3 proteases. Results demonstrated that $C a p-D 3$ dsRNA expression does increase the number of DCP-1-positive cells that lie immediately anterior to the cell exhibiting increased levels of dSRF in the A/P boundary (Fig. 6A). Furthermore, increases in DCP-1 are abrogated when knot dsRNA or UAS-EGFR is expressed in combination with Cap-D3 dsRNA (Fig. 6A,B), although the rescue of cell death is somewhat variable, and discs that exhibit some residual DCP-1 in the pouch also exhibit increased dSRF in the neighboring areas (Fig. 6A, bottom right panel, dorsal side of wing disc). Cell death was largely unaffected in the notum of the majority of these discs (Fig. S10). These findings suggest that CAP-D3 repression of knot in cells anterior to the A/P boundary maintains EGFR activity to promote cell survival.

To determine whether rescue of cell death in CAP-D3-deficient cells was sufficient to prevent the upregulation of dSRF, the baculovirus inhibitor of caspase, p35, was expressed in combination with Cap-D3 dsRNA and discs were again stained for DCP-1 and dSRF. The increased levels of DCP-1 were abrogated, as expected (Fig. 6C). The results however showed that increased levels of dSRF remain, as compared with sca-GAL4-driven Cap-D3 dsRNA expression alone (compare Fig. 6C with 6A). Since CAP-D3 levels and EGFR activity remain low in cells expressing p35 that
A sca-GAL4>UAS-GFP dSRNA, UAS-mRFP

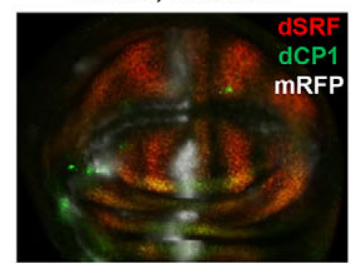

sca-GAL4>UAS-dCAP-D3 dsRNA, UAS-GFP dsRNA

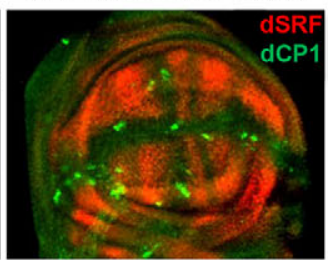

Sca-GAL4>UAS-dCAP-D3
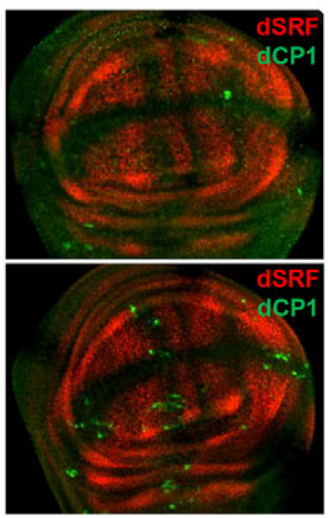

B SCa-GAL4>UAS-dCAP-D
dSRNA, UAS-mRFP

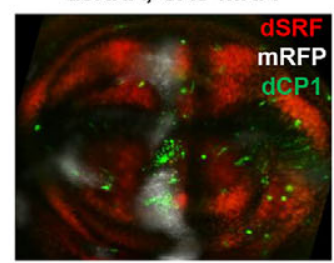

SCa-GAL4>UAS-dCAP-D3 dsRNA, UAS-EGFR

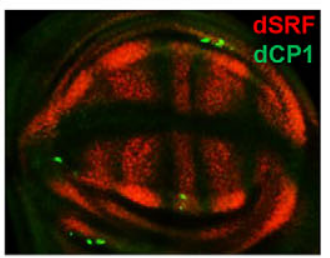

C sca-GAL4>UAS-dCAP-D3 dsRNA, UAS-p35
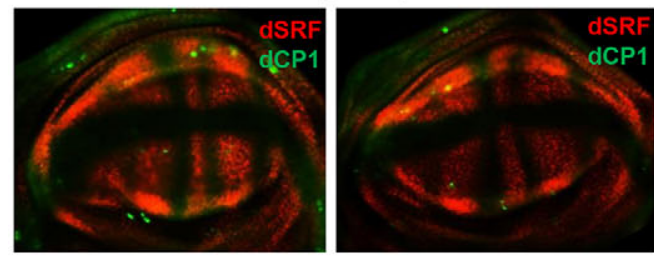

Fig. 6. CAP-D3 deficiency in cells anterior to the A/P boundary results in cell death. (A) Immunostaining for dSRF (red) and DCP-1 (dCP1, green) show variably elevated levels of DCP-1 in cells anterior to the A/P boundary in discs expressing Cap-D3 dsRNA as compared with discs expressing GFP dsRNA (compare middle and leftmost panels). DCP-1 staining in discs expressing both Cap-D3 dsRNA and knot dsRNA more closely resembles that of control discs (compare rightmost and leftmost panels). (B) Immunostaining for dSRF (white) and DCP-1 (green) in wing discs expressing EGFR and Cap-D3 dsRNA (right panel) show decreased levels of DCP-1 in comparison to discs expressing Cap-D3 dsRNA alone (left panel). (C) Immunostaining for dSRF (red) and DCP-1 (green) in wing discs expressing p35 (the baculovirus inhibitor of caspase) and Cap-D3 dsRNA show decreased levels of DCP-1 in comparison to discs expressing Cap-D3 dsRNA alone (compare with B, left panels). Discs shown are representative of three experiments on at least five individual larvae per experiment. 
were rescued from cell death, these data indicate that CAP-D3 and EGFR activity is also necessary for the non-cell-autonomous signaling that occurs between the cells anterior to and the cells within the $\mathrm{A} / \mathrm{P}$ boundary that is responsible for blocking the expression of dSRF.

\section{DISCUSSION CAP-D3/Condensin II-mediated regulation of cell fate in the wing disc}

The Drosophila wing represents an excellent model with which to study signaling pathways and transcriptional programs that influence cell fate decision and, more specifically, the differentiation of cells into vein or intervein. The ACV and PCV begin to develop structurally during the pupal stage, but ACV development is largely influenced by the expression of dSRF and its regulators during the third instar larval stage (Montagne et al., 1996; Roch et al., 1998). The data presented here show that CAP-D3 expression in cells anterior to the A/P boundary (pink anterior cells on the ventral and dorsal side, Fig. 7A) prevents the upregulation of dSRF in a non-cell-autonomous manner in the two neighboring regions positioned between L3 and L4 at the dorsalmost and ventralmost areas of the pouch (Fig. 7A, blue circles). The data suggest that CAP-D3 binds sites surrounding the KN01 enhancer and represses the transcription and expression of knot. This maintains EGFR activity in the cells anterior to the A/P boundary, thus promoting cell survival and facilitating an unidentified EGFRdependent signal that travels to the neighboring $\mathrm{A} / \mathrm{P}$ boundary cell to dampen dSRF expression and block the cell fate decision to become an intervein cell (Fig. 7B). This novel mechanism by which CAP-D3 promotes development of the ACV is the first report of the involvement of a Condensin II subunit in regulating cell fate decisions in vivo. The data presented here show that other Condensin II subunits may play a role in this regulation, as their downregulation in combination with CAP-D3 enhances the loss-ofACV phenotype (Fig. S2). However, since individual knockdown of the other subunits does not cause the phenotype it is possible that either CAP-D3 levels are rate limiting for the proposed pathways or that the dsRNAs used to knock down the other subunits are inefficient. The latter is currently hard to test, given the lack of available antibodies that can be used for this purpose.

\section{Maintenance of EGFR activity by CAP-D3}

EGFR activity is important for the specification of many different cell types throughout fly development. To our knowledge, this is the first report of a Condensin complex functioning to maintain EGFR signaling during development. In an effort to understand whether the ability of CAP-D3 to regulate EGFR activity exists in other cell types, CAP-D3 levels were altered in ovarian follicle cells. It is well known that EGFR activation is required for the establishment of the dorsal/ventral axis of the egg and the embryo (Queenan et al., 1997; Price et al., 1989). Expression of an activated form of EGFR (lambda top) in the follicle cells of the ovary induces a dorsal cell fate in the developing egg, whereas expression of a dominantnegative form of EGFR induces a ventral cell fate. Therefore, to determine whether altering the levels of CAP-D3 would have similar effects, we expressed UAS-GFP-CAP-D3 or Cap-D3 dsRNA in the follicle cells using the T155-GAL4 driver (Hrdlicka et al., 2002) and examined eggs laid by these females. Although we did observe the expected phenotypes caused by overexpression of constitutively active or dominant-negative EGFR, we did not see similar morphological changes due to overexpression or underexpression of CAP-D3 (Fig. S11). These data suggest that the ability of CAP-D3 to promote EGFR activity is not functional in all cells in the fly and might be very tissue specific. It is also possible that CAP-D3 only promotes EGFR activity in tissues where KNOT is expressed and involved in the repression of EGFR.

A major question that develops from these studies is the identity of the signal that is elicited by cells just anterior to the $\mathrm{A} / \mathrm{P}$ boundary and which acts on the cells in the A/P boundary to potentiate cell fate decisions. One possibility is that the EGFR ligand Spitz is responsible for the signaling between the cells, and that in CAP-D3deficient cells there might be a defect in Spitz localization. It was recently shown that the localization of transmembrane Spitz to the apical region of imaginal disc cells renders it incapable of activating EGFR in neighboring cells (Steinhauer et al., 2013). Both overexpression of secreted Spitz and knockdown of Argos, which associates predominantly with Spitz to prevent EGFR signaling (Vinos and Freeman, 2000), rescue the loss-of-ACV phenotype in CAP-D3-deficient cells (Fig. 3C).

Interestingly, cell adhesion can also play a major role in potentiating the EGFR signal from one cell to the next. RAP1, a
A

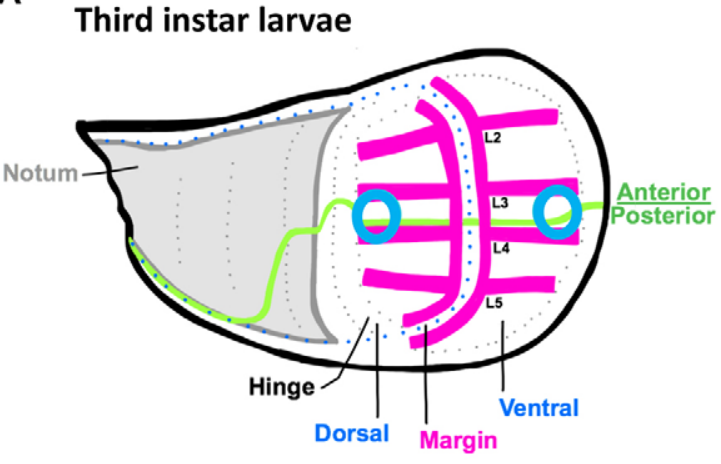

B L3 provein cell ACV progenitor cell

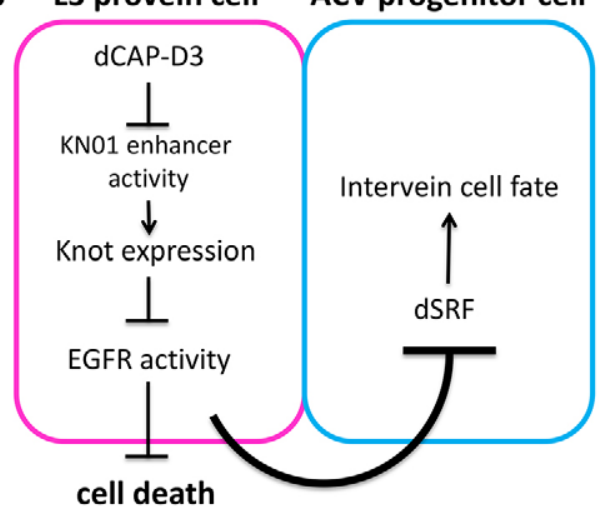

Fig. 7. Model of the mechanism by which CAP-D3 regulates cell fate determination in cells destined to become ACV progenitor cells. (A) Diagram of the third instar larval wing disc. Blue circles represent the regions from which ACV progenitor cells develop and pink lines represent longitudinal vein precursor cells. (B) In the model, CAP-D3 represses knot transcription, potentially by binding to the KN01 enhancer and preventing its ability to activate knot transcription in the L3 provein cells just anterior to the ACV progenitor cells present in the A/P boundary. This repression of knot expression maintains EGFR activity in these cells, preventing cell death and facilitating an EGFR-dependent signal that acts to dampen dSRF expression in neighboring ACV progenitor cells. This keeps dSRF levels balanced with other regulators of cell fate determination, allowing the cells to become ACV. 
small GTPase that regulates DE-cadherin (Shotgun)-mediated cell adhesion, indirectly maintains EGFR signaling in the wing disc through these contacts, and Rapl mutant tissue exhibits planar cell polarity defects and loss of wing vein differentiation (O'Keefe et al., 2009). Although we have not formally tested the idea, it is possible that CAP-D3 deficiency anterior to the $\mathrm{A} / \mathrm{P}$ boundary results in loss of cell-cell adhesion, thus preventing transduction of the EGFR signal into the $\mathrm{A} / \mathrm{P}$ boundary cells.

\section{Potential mechanisms by which CAP-D3 may regulate the KN01 enhancer}

Maintenance of the EGFR signal in cells anterior to the $\mathrm{A} / \mathrm{P}$ boundary seems to rely on the ability of CAP-D3 to repress knot (Fig. 4). Our data suggest that CAP-D3 may repress knot through binding to regions surrounding the KN01 enhancer and maintaining H3K9me3 (Fig. 5). H3K9me3 is found at repressed chromatin, and more recently was shown to be present at 'poised' enhancers in mouse ESCs (Zentner et al., 2011); active enhancers in these cells were found to lose the $\mathrm{H} 3 \mathrm{~K} 9 \mathrm{me} 3$ mark. Given that Condensins are implicated in the regulation of DNA looping in vitro and in Drosophila tissue culture cells (Bazett-Jones et al., 2002; Kimura et al., 1999; Li et al., 2015), it is possible that CAP-D3 regulates looping at the KN01 enhancer to maintain a repressed, yet poised, chromatin state.

An analysis of chromatin marks present in the region, using modENCODE (www.modencode.org; Kharchenko et al., 2011), suggests that a large Polycomb-repressed domain exists over the entire knot locus but, interestingly, that open chromatin appears toward the $3^{\prime}$ end. This suggests that a border between transcriptionally active and repressed chromatin might exist very close to the KN01 enhancer. As Condensin II has been shown to bind to these types of borders and to help to organize topologically associated domains (TADs), it is possible that CAP-D3 binding to KN01 functions in this capacity (Van Bortle et al., 2014; Li et al., 2015). Furthermore, disruption of TADs can alter long-range interactions between enhancers and promoters or other gene regulatory elements, resulting in gene misexpression and serious developmental abnormalities (Lupianez et al., 2015). Future chromosome conformation capture (3C) assays will be necessary to test these ideas.

\section{Broader implications of CAP-D3-mediated knot repression}

KNOT expression is important for Drosophila development in several contexts. It regulates head development (Crozatier et al., 1996, 1999), is important for embryonic muscle cell fate specification (Crozatier and Vincent, 1999; Dubois et al., 2007) and regulates hematopoietic progenitor cell fate (Pennetier et al., 2012; Benmimoun et al., 2015). KNOT is expressed in postmitotic neurons and controls class IV-specific dendritic arbor morphology in the Drosophila peripheral nervous system (Crozatier and Vincent, 2008; Hattori et al., 2013; Jinushi-Nakao et al., 2007). KNOT is also necessary (in combination with other transcription factors) for cell fate specification of specific neurons in the ventral nerve cord and is part of a feed-forward cascade that activates specific terminal differentiation genes in specific neurons (Baumgardt et al., 2007). Overexpression of KNOT causes misexpression of major neuropeptides and drives the cell toward one fate over another (Baumgardt et al., 2009). The question then arises as to whether CAP-D3 might contribute to cell fate specification programs in specific neurons and/or other tissues through its ability to repress knot transcription. The fact that Cap-D3 dsRNA expression mediated by some GAL4 drivers that drive expression in the CNS results in pupal lethality (Table 1), suggests that CAP-D3 might play an important role in the development of specific cells in the CNS and is an idea that needs to be more thoroughly explored.

Recently, it was shown that a high sugar diet promotes O-linked $\mathrm{N}$-acetylglucosamine (O-GlcNAc) transferase (OGT)-mediated OGlcNAc modification of Polyhomeotic, leading to Polycombmediated upregulation of knot in Drosophila pericardial nephrocytes, which are analogous to the mammalian podocyte (Na et al., 2015). Podocytes encircle the glomerular capillaries, and reorganization of their actin cytoskeleton in diabetic patients can lead to albuminuria (Reidy et al., 2014). Upregulation of knot in response to the high sugar diet caused downregulation of the Nephrin-like protein SNS in Drosophila nephrocytes and resulted in phenotypes similar to those seen in patients with diabetic nephropathy ( $\mathrm{Na}$ et al., 2015). The mammalian orthologs of KNOT are the Early B-cell factor (EBF) proteins EBF 1-4 and the $\mathrm{Na}$ et al. (2015) study also showed that nuclear EBF2 levels were increased in both human patients and mouse models of diabetic kidney disease. If the novel pathway that we describe here is conserved in human cells, with CAP-D3 (NCAPD3)/Condensin II mediating repression of EBF transcription factors, then CAP-D3 may represent a novel therapeutic target, the facilitated upregulation of which might help to remedy functional defects in podocytes exhibited by diabetic patients.

\section{MATERIALS AND METHODS}

\section{Drosophila stocks and cell lines}

The following stocks were obtained from the Vienna Drosophila RNAi Library: UAS-Cap-D3-dsRNA ${ }^{G D 913}$, UAS-Cap-H2-dsRNA $A^{K K 103162}$ UAS-SMC2-dsRNA ${ }^{G D 4489}, \quad U A S-S M C 2-d S R N A^{K K 100466}, \quad U A S-S M C 4-$ $d s R N A^{G D 4454}$ and $U A S$-knot-dsRNA $A^{K K 114322}$. The following stocks were obtained from the Bloomington Drosophila Stock Center at Indiana University: $p x[1]$ bs [3],UAS-Egfr $(w[+m c]=U A S-E g f r . B), E g f r$ dominant-negative $(w[+m c]=U A S . E g f r . D N . B)$, argos dsRNA stock $(y[+t 7.7] v[+t 1.8]=$ TRiP.JF03020 $), \quad k n o t \quad$ dsRNA $(y[+t 7.7] v[+t 1.8]$ $=$ TRiP.JF02206), UAS-knot $(w[+m c]=U A S-k n . M)$ and all GAL4 driver stocks. The $k n[1]$ stock was obtained from the Kyoto Drosophila Genetic Resource Center. Other stocks used were: UAS-sKer (Urban et al., 2002), UAS-GrkATC (Queenan et al., 1999), UAS-vn1.1 and UAS-vn1.2 (Schnepp et al., 1998) and $k n[c o l-1]$ (Crozatier and Vincent, 1999). All flies were maintained on standard dextrose medium. All crosses were performed at $25^{\circ} \mathrm{C}$. S2 cells were obtained from the Drosophila Genomics Resource Center at Indiana University (where they are termed S2-DRSC).

\section{Immunofluorescence}

Third instar larval wing discs were dissected in PBS on ice for $15 \mathrm{~min}$ then fixed with $4 \%$ paraformaldehyde and $0.5 \%$ Triton X-100 for 25 min with rocking at room temperature. Discs were washed twice in PBT $(0.1 \%$ Triton $\mathrm{X}-100$ in PBS) plus 1\% BSA (PBT-BSA) before blocking for $2 \mathrm{~h}$ at room temperature in PBT-BSA buffer. Primary antibodies were diluted in PBTBSA and discs were incubated overnight at $4^{\circ} \mathrm{C}$. Discs were washed five times in PBT for $5 \mathrm{~min}$ each at room temperature with rocking. All secondary antibodies were diluted 1:500 in PBT-BSA and incubated with discs for $2 \mathrm{~h}$ at room temperature with rocking. Discs were washed five times in PBT before two 5 min washes in $0.3 \%$ Tween 20 in PBS. Discs were mounted in VectaShield with DAPI (Vector Labs). Imaging was performed on a Zeiss AxioImager Z1 motorized epifluorescence microscope using an MRm CCD camera with a $20 \times$ objective lens. Antibodies are listed in the supplementary Materials and Methods.

\section{RNA in situ hybridization}

Probes were designed as described by Zimmerman et al. (2013) with slight modifications using knot cDNA clone RE03728 (Drosophila Genomics Resource Center at Indiana University). RNA in situ hybridization using 
sense (not shown) and antisense probes was performed as described by $\mathrm{Su}$ et al. (2011) and developed using the Vector Red Alkaline Phosphatase Substrate Kit (Vector Laboratories). Images were taken on a Leica SCN400 slide scanner.

\section{dsRNA treatment and qRT-PCR analysis}

dsRNA treatments and qRT-PCR analyses were performed as described (Schuster et al., 2013). Primers are listed in the supplementary Materials and Methods.

\section{ChIP and ChIP-seq analyses}

ChIP experiments were performed as described (Schuster et al., 2013). DNA used in both ChIP and ChIP-seq experiments was sheared with 500 units of micrococcal nuclease per tube containing nuclei from $5 \times 10^{7}$ cells. $10 \mu \mathrm{g}$ CAP-D3 antibody YZ384 (Longworth et al., 2012) or $10 \mu \mathrm{g}$ IgG antibody was used for immunoprecipitations. Three separate ChIP experiments were performed for CAP-D3 and IgG for both ChIP-qRT-PCR and ChIP-seq. DNA samples used in ChIP-seq were quantitated by qubit (Life Technologies) and ChIP-seq libraries were prepared at the University of Chicago Genomics Core (input 5-10 ng DNA) using the Illumina TruSeq ChIP Sample Prep Kit (IP202-1012). Library quantity and quality was assessed by a Bioanalyzer (Agilent). The pool of libraries was sequenced using the Illumina HiSeq 2500. Data analysis is described in the supplementary Materials and Methods. Highthroughput sequencing data described in this manuscript have been deposited at GEO under accession GSE74413.

\section{Measurement of width of the L3-L4 intervein region}

Adult wings were dissected, mounted in Euparal Mounting Medium (Bioquip Products, 6372A) and then imaged on a Leica DFC3000 G stereo fluorescence microscope. The width of the L3-L4 intervein region, from outer edge to outer edge, was measured using arbitrary units in Adobe Photoshop (1 unit $=0.085 \mathrm{~mm}$ )

\section{Acknowledgements}

We thank Dr Seth Blair for reagents; Dr Michelle Crozatier for fly stocks; and members of the M.S.L. lab for advice and assistance with protocols and design of experiments. Stocks obtained from the Bloomington Drosophila Stock Center [NIH P40OD018537] and the Vienna Drosophila Research Center were used in this study. Drosophila S2 cells were obtained from the Drosophila Genomics Resource Center at Indiana University [NIH 2P40OD010949-10A1].

\section{Competing interests}

The authors declare no competing or financial interests.

\section{Author contributions}

L.R.K., E.C.P., A.T.S., K.D., K.S., J.J.L., J.A.M. and M.S.L. performed experiments. M.E.L. performed statistical analyses of ChIP-seq data. L.R.K., E.C.P., M.E.L. and M.S.L. wrote the manuscript. J.A.M. and A.W.M. provided reagents and edited the manuscript.

\section{Funding}

M.S.L. is supported by a research grant from the National Institutes of Health [R01GM102400]. Deposited in PMC for immediate release.

\section{Data availability}

ChIP-seq data have been deposited at Gene Expression Omnibus under accession number GSE74413 (http://www.ncbi.nlm.nih.gov/geo/query/acc.cgi? token=whybyomgrdextsv\&acc=GSE74413)

\section{Supplementary information}

Supplementary information available online at

http://dev.biologists.org/lookup/doi/10.1242/dev.133686.supplemental

\section{References}

Bauer, C. R., Hartl, T. A. and Bosco, G. (2012). Condensin II promotes the formation of chromosome territories by inducing axial compaction of polyploid interphase chromosomes. PLoS Genet. 8, e1002873.

Baumgardt, M., Miguel-Aliaga, I., Karlsson, D., Ekman, H. and Thor, S. (2007) Specification of neuronal identities by feedforward combinatorial coding. PLOS Biol. 5, e37.

Baumgardt, M., Karlsson, D., Terriente, J., Diaz-Benjumea, F. J. and Thor, S. (2009). Neuronal subtype specification within a lineage by opposing temporal feed-forward loops. Cell 139, 969-982.
Bazett-Jones, D. P., Kimura, K. and Hirano, T. (2002). Efficient supercoiling of DNA by a single condensin complex as revealed by electron spectroscopic imaging. Mol. Cell 9, 1183-1190.

Benmimoun, B., Polesello, C., Haenlin, M. and Waltzer, L. (2015). The EBF transcription factor Collier directly promotes Drosophila blood cell progenito maintenance independently of the niche. Proc. Natl. Acad. Sci. USA 112 9052-9057.

Bergmann, A., Agapite, J., McCall, K. and Steller, H. (1998). The Drosophila gene hid is a direct molecular target of Ras-dependent survival signaling. Cell 95 331-341.

Biehs, B., Sturtevant, M. A. and Bier, E. (1998). Boundaries in the Drosophila wing imaginal disc organize vein-specific genetic programs. Development 125 4245-4257.

Conley, C. A., Silburn, R., Singer, M. A., Ralston, A., Rohwer-Nutter, D., Olson, D. J., Gelbart, W. and Blair, S. S. (2000). Crossveinless 2 contains cysteine-rich domains and is required for high levels of BMP-like activity during the formation of the cross veins in Drosophila. Development 127, 3947-3959.

Crozatier, M. and Vincent, A. (1999). Requirement for the Drosophila COE transcription factor Collier in formation of an embryonic muscle: transcriptional response to notch signalling. Development 126, 1495-1504.

Crozatier, M. and Vincent, A. (2008). Control of multidendritic neuron differentiation in Drosophila: the role of Collier. Dev. Biol. 315, 232-242.

Crozatier, M., Valle, D., Dubois, L., Ibnsouda, S. and Vincent, A. (1996). Collier, a novel regulator of Drosophila head development, is expressed in a single mitotic domain. Curr. Biol. 6, 707-718.

Crozatier, M., Valle, D., Dubois, L., Ibnsouda, S. and Vincent, A. (1999). Head versus trunk patterning in the Drosophila embryo; collier requirement for formation of the intercalary segment. Development 126, 4385-4394.

De Celis, J. F. (1997). Expression and function of decapentaplegic and thick veins during the differentiation of the veins in the Drosophila wing. Development 124, 1007-1018.

De Celis, J. F., Bray, S. and Garcia-Bellido, A. (1997). Notch signalling regulates veinlet expression and establishes boundaries between veins and interveins in the Drosophila wing. Development 124, 1919-1928.

Diaz-Benjumea, F. J. and Garcia-Bellido, A. (1990). Behaviour of cells mutant for an EGF receptor homologue of Drosophila in genetic mosaics. Proc. R. Soc. B Biol. Sci. 242, 36-44.

Donlea, J. M., Ramanan, N. and Shaw, P. J. (2009). Use-dependent plasticity in clock neurons regulates sleep need in Drosophila. Science 324, 105-108.

Doroquez, D. B. and Rebay, I. (2006). Signal integration during development mechanisms of EGFR and Notch pathway function and cross-talk. Crit. Rev. Biochem. Mol. Biol. 41, 339-385.

Dowen, J. M., Bilodeau, S., Orlando, D. A., Hübner, M. R., Abraham, B. J., Spector, D. L. and Young, R. A. (2013). Multiple structural maintenance of chromosome complexes at transcriptional regulatory elements. Stem Cell Rep. 1 371-378.

Dubois, L., Enriquez, J., Daburon, V., Crozet, F., Lebreton, G., Crozatier, M. and Vincent, A. (2007). Collier transcription in a single Drosophila muscle lineage: the combinatorial control of muscle identity. Development 134, 4347-4355.

Fristrom, D., Gotwals, P., Eaton, S., Kornberg, T. B., Sturtevant, M., Bier, E. and Fristrom, J. W. (1994). Blistered: a gene required for vein/intervein formation in wings of Drosophila. Development 120, 2661-2671.

Gabay, L., Seger, R. and Shilo, B.-Z. (1997). In situ activation pattern of Drosophila EGF receptor pathway during development. Science 277, 1103-1106.

Garcia-Bellido, A. and De Celis, J. F. (1992). Developmental genetics of the venation pattern of Drosophila. Annu. Rev. Genet. 26, 277-304.

Guichard, A., Biehs, B., Sturtevant, M. A., Wickline, L., Chacko, J., Howard, K. and Bier, E. (1999). rhomboid and Star interact synergistically to promote EGFR MAPK signaling during Drosophila wing vein development. Development 126 2663-2676.

Hartl, T. A., Smith, H. F. and Bosco, G. (2008). Chromosome alignment and transvection are antagonized by condensin II. Science 322, 1384-1387.

Hattori, Y., Usui, T., Satoh, D., Moriyama, S., Shimono, K., Itoh, T., Shirahige, K and Uemura, T. (2013). Sensory-neuron subtype-specific transcriptional programs controlling dendrite morphogenesis: genome-wide analysis of Abrupt and Knot/Collier. Dev. Cell 27, 530-544.

Hersh, B. M. and Carroll, S. B. (2005). Direct regulation of knot gene expression by Ultrabithorax and the evolution of cis-regulatory elements in Drosophila. Development 132, 1567-1577.

Hrdlicka, L., Gibson, M., Kiger, A., Micchelli, C., Schober, M., Schöck, F. and Perrimon, N. (2002). Analysis of twenty-four Gal4 lines in Drosophila melanogaster. Genesis 34, 51-57.

Jinushi-Nakao, S., Arvind, R., Amikura, R., Kinameri, E., Liu, A. W. and Moore, A. W. (2007). Knot/Collier and cut control different aspects of dendrite cytoskeleton and synergize to define final arbor shape. Neuron 56, 963-978.

Joyce, E. F., Williams, B. R., Xie, T. and Wu, C.-T. (2012). Identification of genes that promote or antagonize somatic homolog pairing using a high-throughput FISH-based screen. PLoS Genet. 8, e1002667.

Kharchenko, P. V., Alekseyenko, A. A., Schwartz, Y. B., Minoda, A., Riddle, N. C., Ernst, J., Sabo, P. J., Larschan, E., Gorchakov, A. A., Gu, T. et al. (2011). 
Comprehensive analysis of the chromatin landscape in Drosophila melanogaster. Nature 471, 480-485.

Kimura, K., Rybenkov, V. V., Crisona, N. J., Hirano, T. and Cozzarelli, N. R. (1999). 13S condensin actively reconfigures DNA by introducing global positive writhe: implications for chromosome condensation. Cell 98, 239-248.

Kranz, A.-L., Jiao, C.-Y., Winterkorn, L. H., Albritton, S. E., Kramer, M. and Ercan, S. (2013). Genome-wide analysis of condensin binding in Caenorhabditis elegans. Genome Biol. 14, R112.

Li, L., Lyu, X., Hou, C., Takenaka, N., Nguyen, H. Q., Ong, C.-T., Cubeñas-Potts, C., Hu, M., Lei, E. P., Bosco, G. et al. (2015). Widespread rearrangement of 3D chromatin organization underlies polycomb-mediated stress-induced silencing Mol. Cell 58, 216-231.

Longworth, M. S. and Dyson, N. J. (2010). pRb, a local chromatin organizer with global possibilities. Chromosoma 119, 1-11.

Longworth, M. S., Walker, J. A., Anderssen, E., Moon, N.-S., Gladden, A., Heck, M. M. S., Ramaswamy, S. and Dyson, N. J. (2012). A shared role for RBF1 and dCAP-D 3 in the regulation of transcription with consequences for innate immunity. PLoS Genet. 8, e1002618.

Lupianez, D. G., Kraft, K., Heinrich, V., Krawitz, P., Brancati, F., Klopocki, E., Horn, D., Kayserili, H., Opitz, J. M., Laxova, R. et al. (2015). Disruptions of topological chromatin domains cause pathogenic rewiring of gene-enhancer interactions. Cell 161, 1012-1025

Martin-Blanco, E., Roch, F., Noll, E., Baonza, A., Duffy, J. B. and Perrimon, N. (1999). A temporal switch in DER signaling controls the specification and differentiation of veins and interveins in the Drosophila wing. Development 126 5739-5747.

Matakatsu, H., Tadokoro, R., Gamo, S. and Hayashi, S. (1999). Repression of the wing vein development in Drosophila by the nuclear matrix protein plexus. Development 126, 5207-5216.

McKay, D. J. and Lieb, J. D. (2013). A common set of DNA regulatory elements shapes Drosophila appendages. Dev. Cell 27, 306-318.

Mlodzik, M., Baker, N. E. and Rubin, G. M. (1990). Isolation and expression of scabrous, a gene regulating neurogenesis in Drosophila. Genes Dev. 4 , 1848-1861.

Mohler, J., Seecoomar, M., Agarwal, S., Bier, E. and Hsai, J. (2000). Activation of knot $(\mathrm{kn})$ specifies the 3-4 intervein region in the Drosophila wing. Development $127,55-63$

Montagne, J., Groppe, J., Guillemin, K., Krasnow, M. A., Gehring, W. J. and Affolter, M. (1996). The Drosophila Serum Response Factor gene is required fo the formation of intervein tissue of the wing and is allelic to blistered. Development $122,2589-2597$

Na, J., Sweetwyne, M. T., Park, A. S. D., Susztak, K. and Cagan, R. L. (2015). Diet-induced podocyte dysfunction in Drosophila and mammals. Cell Rep. 12 636-647.

Nussbaumer, U., Halder, G., Groppe, J., Affolter, M. and Montagne, J. (2000). Expression of the blistered/DSRF gene is controlled by different morphogens during Drosophila trachea and wing development. Mech. Dev. 96, 27-36.

O'Keefe, D. D., Gonzalez-Niño, E., Burnett, M., Dylla, L., Lambeth, S. M., Licon, E., Amesoli, C., Edgar, B. A. and Curtiss, J. (2009). Rap1 maintains adhesion between cells to affect Egfr signaling and planar cell polarity in Drosophila. Dev. Biol. 333, 143-160.

Pennetier, D., Oyallon, J., Morin-Poulard, I., Dejean, S., Vincent, A. and Crozatier, M. (2012). Size control of the Drosophila hematopoietic niche by bone morphogenetic protein signaling reveals parallels with mammals. Proc. Natl. Acad. Sci. USA 109, 3389-3394.

Posakony, L. G., Raftery, L. A. and Gelbart, W. M. (1990). Wing formation in Drosophila melanogaster requires decapentaplegic gene function along the anterior-posterior compartment boundary. Mech. Dev. 33, 69-82.

Price, J. V., Clifford, R. J. and Schüpbach, T. (1989). The maternal ventralizing locus torpedo is allelic to faint little ball, an embryonic lethal, and encodes the Drosophila EGF receptor homolog. Cell 56, 1085-1092.

Queenan, A. M., Ghabrial, A. and Schupbach, T. (1997). Ectopic activation of torpedo/Egfr, a Drosophila receptor tyrosine kinase, dorsalizes both the eggshell and the embryo. Development 124, 3871-3880.
Queenan, A. M., Barcelo, G., Van Buskirk, C. and Schüpbach, T. (1999). The transmembrane region of Gurken is not required for biological activity, but is necessary for transport to the oocyte membrane in Drosophila. Mech. Dev. $\mathbf{8 9}$ 35-42.

Rawlings, J. S., Gatzka, M., Thomas, P. G. and Ihle, J. N. (2011). Chromatin condensation via the condensin II complex is required for peripheral T-cel quiescence. EMBO J. 30, 263-276.

Reidy, K., Kang, H. M., Hostetter, T. and Susztak, K. (2014). Molecular mechanisms of diabetic kidney disease. J. Clin. Invest. 124, 2333-2340.

Roch, F., Baonza, A., Martin-Blanco, E. and Garcia-Bellido, A. (1998). Genetic interactions and cell behaviour in blistered mutants during proliferation and differentiation of the Drosophila wing. Development 125, 1823-1832.

Schnepp, B., Grumbling, G., Donaldson, T. and Simcox, A. (1996). Vein is a novel component in the Drosophila epidermal growth factor receptor pathway with similarity to the neuregulins. Genes Dev. 10, 2302-2313.

Schnepp, B., Donaldson, T., Grumbling, G., Ostrowski, S., Schweitzer, R. Shilo, B.-Z. and Simcox, A. (1998). EGF domain swap converts a drosophila EGF receptor activator into an inhibitor. Genes Dev. 12, 908-913.

Schuster, A. T., Sarvepalli, K., Murphy, E. A. and Longworth, M. S. (2013) Condensin II subunit dCAP-D3 restricts retrotransposon mobilization in Drosophila somatic cells. PLoS Genet. 9, e1003879.

Schweitzer, R., Shaharabany, M., Seger, R. and Shilo, B. Z. (1995). Secreted Spitz triggers the DER signaling pathway and is a limiting component in embryonic ventral ectoderm determination. Genes Dev. 9, 1518-1529.

Singson, A., Leviten, M. W., Bang, A. G., Hua, X. H. and Posakony, J. W. (1994) Direct downstream targets of proneural activators in the imaginal disc include genes involved in lateral inhibitory signaling. Genes Dev. 8, 2058-2071.

Steinhauer, J., Liu, H. H., Miller, E. and Treisman, J. E. (2013). Trafficking of the EGFR ligand Spitz regulates its signaling activity in polarized tissues. J. Cell Sci. 126, 4469-4478.

Sturtevant, M. A. and Bier, E. (1995). Analysis of the genetic hierarchy guiding wing vein development in Drosophila. Development 121, 785-801.

Sturtevant, M. A., Roark, M. and Bier, E. (1993). The Drosophila rhomboid gene mediates the localized formation of wing veins and interacts genetically with components of the EGF-R signaling pathway. Genes Dev. 7, 961-973.

Su, Y., Ospina, J. K., Zhang, J., Michelson, A. P., Schoen, A. M. and Zhu, A. J. (2011). Sequential phosphorylation of smoothened transduces graded hedgehog signaling. Sci. Signal. 4, ra43.

Urban, S., Lee, J. R. and Freeman, M. (2002). A family of Rhomboid intramembrane proteases activates all Drosophila membrane-tethered EGF ligands. EMBO J. 21, 4277-4286.

Valentine, M., Hogan, J. and Collier, S. (2014). The Drosophila Chmp1 protein determines wing cell fate through regulation of epidermal growth factor receptor signaling. Dev. Dyn. 243, 977-987.

Van Bortle, K., Nichols, M. H., Li, L., Ong, C.-T., Takenaka, N., Qin, Z. S. and Corces, V. G. (2014). Insulator function and topological domain border strength scale with architectural protein occupancy. Genome Biol. 15, R82

Vervoort, M. (2000). hedgehog and wing development in Drosophila: a morphogen at work? Bioessays 22, 460-468

Vervoort, M., Crozatier, M., Valle, D. and Vincent, A. (1999). The COE transcription factor Collier is a mediator of short-range Hedgehog-induced patterning of the Drosophila wing. Curr. Biol. 9, 632-639.

Vinos, J. and Freeman, M. (2000). Evidence that Argos is an antagonistic ligand of the EGF receptor. Oncogene 19, 3560-3562.

Waddington, C. H. (1940). The genetic control of wing development in Drosophila. J. Genet. 41, 75-113.

Xu, Y., Leung, C. G., Lee, D. C., Kennedy, B. K. and Crispino, J. D. (2006). MTB, the murine homolog of condensin II subunit CAP-G2, represses transcription and promotes erythroid cell differentiation. Leukemia 20, 1261-1269.

Zentner, G. E., Tesar, P. J. and Scacheri, P. C. (2011). Epigenetic signatures distinguish multiple classes of enhancers with distinct cellular functions. Genome Res. 21, 1273-1283.

Zimmerman, S. G., Peters, N. C., Altaras, A. E. and Berg, C. A. (2013). Optimized RNA ISH, RNA FISH and protein-RNA double labeling (IF/FISH) in Drosophila ovaries. Nat. Protoc. 8, 2158-2179. 